\title{
Sicilia 1. Resuttano. I canti dei contadini
}

Enregistrements (1972 et 1995) rassemblés par Ignazio Macchiarella. 1995

\section{Anne-Florence Borneuf}

\section{(2) OpenEdition}

\section{Journals}

Édition électronique

URL : http://journals.openedition.org/ethnomusicologie/1292

ISSN : 2235-7688

\section{Éditeur}

ADEM - Ateliers d'ethnomusicologie

Édition imprimée

Date de publication : 1 décembre 1996

Pagination : 339-340

ISBN : 978-2-8257-0559-9

ISSN : 1662-372X

\section{Référence électronique}

Anne-Florence Borneuf, « Sicilia 1. Resuttano. I canti dei contadini », Cahiers d'ethnomusicologie [En ligne], 9 | 1996, mis en ligne le 05 janvier 2012, consulté le 22 avril 2019. URL : http:// journals.openedition.org/ethnomusicologie/1292

Ce document a été généré automatiquement le 22 avril 2019.

Tous droits réservés 


\section{Sicilia 1. Resuttano. I canti dei contadini}

Enregistrements (1972 et 1995) rassemblés par Ignazio Macchiarella. 1995

\section{Anne-Florence Borneuf}

\section{RÉFÉRENCE}

Sicilia 1. Resuttano. I canti dei contadini. Enregistrements (1972 et 1995) rassemblés par Ignazio Macchiarella. Texte et transcriptions d'Ignazio Macchiarella. Notice de 28 pages en italien, anglais et français. 1 CD Nota/ Micromedia CD 2.21, 1995. Durée totale : 59'. [coll. « Musica e Memoria. Repertori di tradizione orale »].

\section{NOTE DE L'ÉDITEUR}

Rubrique réalisée en collaboration avec le Laboratoire d'ethnomusicologie, UMR 9957 du CNRS, et la Phonothèque du Musée de l'Homme à Paris (coordinateur : Pribislav Pitoëff), notamment à partir de ses acquisitions récentes.

1 C'est à Resuttano, un village situé en plein cœur de la Sicile, que cette monographie musicale est consacrée. Outre les «chants des paysans » annoncés par le titre, le disque propose des répertoires religieux de femmes et des musiques pour banda (ensemble d'instruments à vent et percussion).

2 L'auteur - lui-même sicilien - découvrit les chants des paysans de Resuttano dans les archives du Folkstudio de Palerme. L'émission vocale caractéristique, la richesse ornementale et l'alternance de deux textes différents dans le même chant l'intriguèrent. Mais c'est seulement quinze ans plus tard qu'il eut l'occasion de se rendre sur le terrain.

Or, en quelques années, le monde paysan a énormément évolué. Cette évolution - tant dans le domaine technique que dans le domaine social - a entraîné la disparition de pans entiers de la culture paysanne et, par conséquent, celle des manifestations sonores qui lui étaient liées. Quelques chants sont cependant restés dans les mémoires ou ont pu être 
« recyclés » dans de nouvelles fonctions. C'est donc à la fixation, à la restitution, voire à la reconstruction de cette mémoire, avant sa complète disparition, que s'applique une grande partie de ce disque.

4 De fait, on peut reprocher à certaines plages un manque de conviction musicale, une voix affadie, un déséquilibre flagrant (entre soliste et chœur notamment). Ceci est sensible en particulier dans le chant responsorial de la plage 10, qui, en raison de sa reconstitution récente par le soliste et le chœur, supporte mal la confrontation avec une publication antérieure ${ }^{1}$.

5 Le but ici, n'est pas d'engager une polémique sur la démarche qui a prévalu à la conception de ce disque, mais plutôt de saisir les informations qu'apporte cette publication en tant que résultat d'une recherche monographique sur les répertoires passés et présents - d'un village. Différents corpus sont ainsi regroupés de façon didactique: sirbie (chants exécutés par deux voix masculines alternées avec accompagnement de guimbarde), chants de travail, chants de la semaine sainte, chants monodiques narratifs, rosaires et chants religieux féminins, musiques de fête (fanfare).

$6 \quad$ Les sirbie (plages 1-6)présentent un grand intérêt, tant du point de vue du traitement du texte que de celui de la technique d'ornementation - superbement illustrée dans la première plage ${ }^{2}$ - basée sur l'emprunt intermittent et bref à différents modes d'émission vocale. Parmi les chants de travail, les monodies de battage du blé (plages 7-9) où la voix est projetée dans un registre aigu, étroit et tendu méritent l'attention de l'auditeur.

7 Ainsi que le signale Ignazio Macchiarella, certains des répertoires proposés ici ne sont que rarement intégrés dans les publications discographiques; c'est le cas par exemple des rosaires en antiphonie et des chants religieux féminins (plages 16-19) qui représentent un énorme corpus, vivant et encore à étudier, mais l'auteur, après avoir souligné cette situation, ne propose malheureusement aucune piste d'analyse.

Le livret (treize pages en italien, quatre en anglais, cinq en français) situe chacun des répertoires dans leur contexte (passé et présent), il fournit des indications d'ordre musicologique et donne une transcription de la majorité des textes chantés en dialecte (l'absence de traduction, au moins en italien, est cependant à regretter). En ce qui concerne la version française, l'aplatissement de la pensée de l'auteur, ainsi que l'utilisation de termes obscurs («motif verbal » au lieu de «texte »...) ou peu appropriés (" octave " au lieu de "huitain ", "cadence très embellie » au lieu de "cadence très ornementée »...) sont à regretter car ils rendent plus opaque encore une traduction déjà limitée à un résumé de la notice en italien.

9 En conclusion, la réalisation de ce disque confirme le sérieux qu'Ignazio Macchiarella a démontré dans d'autres publications ${ }^{3}$. Le parti pris de publier des enregistrements faisant (quasi) exclusivement appel à la mémoire de chanteurs aujourd'hui âgés est concevable, mais sur le plan esthétique, l'auditeur reste globalement sur sa faim, et ceci d'autant plus que le " petit bijou » du disque (la première plage) est un document tiré d'archives qui avaient sans doute beaucoup plus qu'une unique plage à nous livrer. 


\section{NOTES}

1. Ce même chant, a en effet été enregistré à Resuttano par E. Guggino et G. Pagano en 1972. L'auteur en signale la publication dans le disque Musiche e canti popolari siciliani. Canti del lavoro vol. 1, 33 t., Albatros VPA 8206, 1972 ; ces enregistrements ont également fait l'objet d'une édition américaine (Folk Music and Songs of Sicily, 33 t., Lyrichord, LLST 7333).

2. Il s'agit d'un des enregistrements réalisés par Elsa Guggino et archivés au Folkstudio de Palerme.

3. On signalera en particulier deux autres monographies discographiques consacrées aux chants de la Semaine Sainte dans les villages de Montedoroet Mussomeli : I 'lamenti' della Settimana Santa di Montedoro, 33 t., Albatros VPA 8488, 1987 et I 'lamenti' di Mussomeli, 33 t., Albatros VPA 8492, 1989. Toujours dans le domaine des chants de la Semaine Sainte, l'auteur a également publié une étude musicologique des répertoires de Sicile et de Sardaigne : Il falsobordone fra tradizione orale e tradizione scritta, Libreria Musicale Italiana Editrice, Lucca, (s. d.). 